\title{
Assessment of Diastolic Dysfunction in Patients with Atrial Fibrillation
}

Mohsen Ali Mahmoud Salama ${ }^{1}$ MD, Wael Mohammed Attia ${ }^{1}$ MD, Mohamed Abdelaziz Mohamed ${ }^{1 *}$ M.Sc.

*CorrespondingAuthor: Mohamed Abdelaziz Mohamed muhamd.zizo@hotmail.com Received for publication January 26, 2020; accepted April 11, 2020; Published on line April 11, 2020.

\section{Copyright 2020 The Authors published by Al-Azhar University, Faculty of Medicine, Cairo, Egypt. All rights reserved. This an open- access article distributed under the legal terms, where it is permissible to download and share the work provided it is properly cited. The work cannot be changed in anyway or used commercially. \\ doi:10.21608/aimj.2020.22090 1108 \\ ${ }^{1}$ Department of Cardiology ,Faculty of Medicine, Al-Azhar University Cairo, Egypt.}

\begin{abstract}
Background: Atrial fibrillation $(\mathrm{AF})$ is the most frequently encountered arrhythmia in clinical practice. Detection of concurrent diastolic dysfunction (DD) may be beneficial in patients with persistent AF. So the aim of the study was to evaluate the best echocardiographic parameter to assess diastolic function in patients with $\mathrm{AF}$.
\end{abstract}

Patients and Methods: The study population consisted of $80 \mathrm{AF}$ patients who were candidate for diagnostic coronary angiography or PCI. During the invasive procedure, the left ventricular end diastolic pressure (LVEDP) was measured then all patients underwent routine clinical and appropriate mitral flow and tissue Doppler velocities as well as standard echocardiographic measurements were obtained within 24 hours of the procedure.

Results: The patients were classified into 2 groups, group A included 40 patients with LVEDP $<16 \mathrm{mmHg}$ and group B included 40 patients with LVEDP $\geq 16 \mathrm{mmHg}$ and found that E/e' average was the independent predictor of LVEDP> $16 \mathrm{mmHg}$ with OR 1.4 and p-value $<0.05 . \mathrm{E} / \mathrm{e}^{\prime}$ average sensitivity was $70 \%$, specificity was $95 \%$, AUORC was .88 , Positive predictive value $93 \%$ and $p$ value was $<0.001$.there was about $74 \%$ concordance between invasive and echocardiographic methods for E/e' average.

Conclusion: E/E' ratio is single best independent predictor of LVEDP with highest sensitivity and specificity when compared with other echocardiographic parameters.

Key words: Atrial Fibrillation, Diastolic Dysfunction

Disclosure: The authors have no financial interest to declare in relation to the content of this article. The Article Processing Charge was paid for by the authors.

\section{INTRODUCTION}

Atrial fibrillation (AF) is an increasingly common heart rhythm disturbance that leads to frequent hospital admissions, heart failure, stroke, and higher mortality ${ }^{1}$. There is a close relationship between AF and heart failure, with numerous risk factors common to both conditions, and shared pathophysiology in patients with both reduce ${ }^{2}$ and preserved $^{3}$ left ventricular ejection fraction (LVEF). Depending on the type of $\mathrm{AF}$, the rate of prevalent heart failure is between $33 \%$ and $56 \%$, hence clinicians treating patients with $\mathrm{AF}$ need reliable information on both systolic and diastolic left ventricular (LV) function ${ }^{4}$.
Diastolic dysfunction shares many common risk factors with $\mathrm{AF}$, including age, hypertension, obesity $^{5}$ and diabetes ${ }^{6}$ Like AF, diastolic dysfunction increases with age ${ }^{7}$.The loss of synchronized atrial contraction and altered left atrial pressure is likely to affect the reproducibility of echocardiographic measurements in AF. Factors that have been implicated include the ratio of proceeding to prepreceding cycle length and heart rate during image acquisition.

\section{PATIENTS AND METHODS}

The study was designed as a prospective observational study. All the Participants were informed of its objectives before the study. The study 
included 80 patients with permanent $\mathrm{AF}$ documented with 12 lead ECG with single lead rhythm strip who were candidate for diagnostic coronary angiography or PCI at National heart institute Hospital - Cairo Egypt between May 2018 and April 2019. Patients with paroxysmal AF, significant valvular disease i.e. more than mild stenosis or reguge, prosthetic valve or mitral valve ring or poor echogenic window were excluded from the study.

During the diagnostic coronary angiography or PCI, LVEDP was measured. Within 24 hours, history taking with special emphasis on: Risk factors (Age, gender, diabetes, hypertension, smoking, dyslipidemia, family history).History of coronary artery syndromes (CAD), complete clinical examination and transthoracic echocardiography was performed .Recordings and calculations of different cardiac chambers and ejection fractions were made according to the recommendations of the American Society of Echocardiography ${ }^{8}$. Examination was performed with Philips IE33. Dedicated cardiac probe was positioned in standard parasternal and apical position and appropriate long and short axis parasternal as well as 4 and 2 chambers apical views were obtained. Ejection fraction was calculated using modified Simpson method. Mitral flow velocity was measured with a pulse wave Doppler and sample volume positioned at the tip of mitral leaflets Peak early (E) transmittal flow velocities and deceleration time were obtained. Tissue Doppler measurements were recorded from apical 4 chamber view. Pulsed wave Doppler was measured with $5 \mathrm{~mm}$ sample volume positioned at junction of mitral annulus and adjacent septal and lateral myocardium. Myocardial early (E') diastolic velocities were measured and average $\left(E / E^{\prime}\right)$ ratios were immediately calculated. Median of 5 consecutive complexes was calculated for all obtained measurements. Left atrial volume index (LAVI) was calculated using biplane arealength method from apical 4 and apical 2 chamber views ${ }^{8}$. Tricuspid regurge (TR) velocity was obtained from color guided CW Doppler from 4 chamber view.

Statistical analysis:

Data were analyzed using Statistical Program for Social Science (SPSS)

Version 25.0 for windows (SPSS Inc., Chicago, IL, USA), NCSS 12 for

Windows (NCSS LCC, Kaysville, UT, USA).

Quantitative data were expressed as mean \pm standard deviation (SD).

Median and inter-quartile range (IQR) were also calculated for quantitative data. Qualitative data were expressed as frequency and percentage.

\section{RESULTS}

The patients were divided into two groups according to the LVEDP, Group A: 40 patients with LVEDP < $16 \mathrm{mmHg}$, This group included 40 patients, 22 patients were male $(55 \%)$ while theremaining18 patients were female (45\%) and Group B: 40 patients with LVEDP $\geq 16 \mathrm{mmHg}$, This group included 40 patients, 24 patients were male $(60 \%)$ while the Remaining 16 patients were female $(40 \%)$.

Baseline clinical characteristics: are presented in (Figure 1) showing no statistically significant differences between both groups regarding the baseline characteristics except the hypertension and ischemic heart disease(IHD) which had statistically significant differences between both groups. ( $P$ value $=0.025$ and 0.036 respectively)

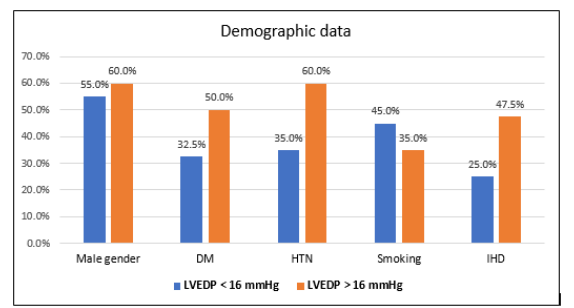

Fig. 1: Baseline clinical characteristics of both groups.

Echocardiographic data:

$\mathrm{EF}(\%)$ : The mean Ejection fraction values 61.0 $\pm 6.9 \%$ were higher in group A than group B $52.7 \pm 7.7$. There was statistically significant difference between the two groups with the $\mathrm{P}$ value $<0.05$. MV deceleration time $(\mathrm{m}-\mathrm{sec})$ : The MV deceleration time median range was111 (100 - 134) in group A, $111(71$ - 142) in group B. There was no statistically significant difference between the two groups with the $\mathrm{P}$ value $>0.05$. E/e' septal: it was $9.7(7.6-10.8)$ in group A, versus $14.9(11.7$ - 22.5) in group B. There was statistically significant difference between the two groups with the $\mathrm{P}$ value $<0.05$. E/e' lateral: its median range was 8.9 (6.9 $10.5)$ in group A, versus $14.5(12.9-19.6)$ in group B. There was statistically significant difference between the two groups with the $\mathrm{P}$ value $<0.05$. E/e' average: its median range was $9.4(7.2-10.5)$ in group A, versus 14.9(12.0 21.8) in group B. There was statistically significant difference between the two groups with the $\mathrm{P}$ value $<0.05$. LAVI $(\mathrm{ml} / \mathrm{m} 2)$ : LAVI median range was $15.6(13.3-18.9)$ in group A, versus23.4 (19.5 - 33.0) in group B. There was statistically significant difference between the two groups with the $\mathrm{P}$ value $<0.05$. TR velocity $(\mathrm{m} / \mathrm{s})$ : The TR velocity median range was 1.5 $(1.2-2.1)$ in group A, versus $2.4(1.9-3.0)$ in group B. There was statistically significant difference between the two groups with the $\mathrm{P}$ value $<0.05$.

As presented in (Figure 2), E/e' average sensitivity was $70 \%$, specificity was $95 \%$, AUORC was .88, Positive predictive value 93\% and $\mathrm{p}$ value was $<0.001$, EF sensitivity $65 \%$ and specificity $80 \%$, LAVI sensitivity $20 \%$ and specificity $100 \%$, TR velocity sensitivity $35 \%$ and specificity $80 \%$. 


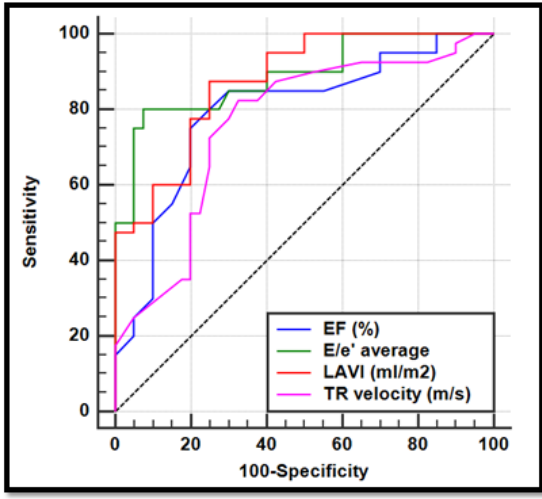

Fig. 2: The predictive performance of echo parameters for LVEDP> $16 \mathrm{mmHg}$; ROC curve analysis

As presented in (Figure 3,4,5) There was about 74\% concordance between invasive and echocardiographic methods for E/e' average, $47 \%$ concordance for LAVI and $47 \%$ concordance for TR velocity.

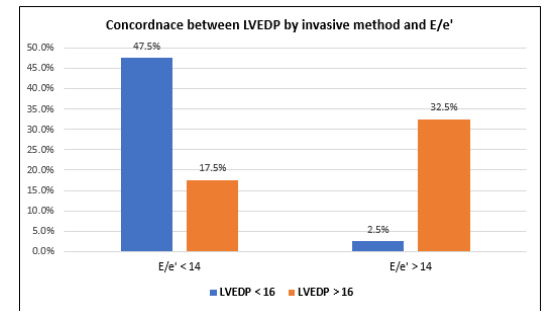

Fig. 3: Concordance between invasive LVEDP and $\mathrm{E} / \mathrm{e}$ '.

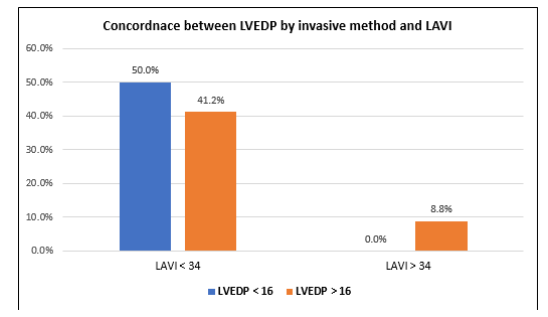

Fig. 4: Concordance between invasive LVEDP and LAVI.

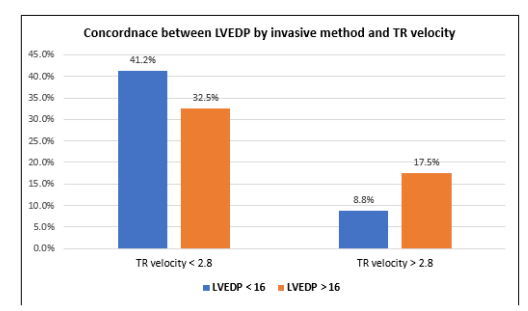

Fig. 5: Concordance between invasive LVEDP and TR velocity.

\section{DISCUSSION}

The results of this study showed that the mean Ejection fraction values were higher in group A more than group B. There was statistically significant difference between the two groups with the $\mathrm{P}$ value < 0.05. This was comparable with Kotecha, et al., ${ }^{9}$ study which reviewed 32 studies from 3066 search results (1968 patients with AF) with mean LVEF 53\% $( \pm 10 \%)$ and found that reduced EF was associated with elevated filling pressure.

In our study, there was no statistically significant difference between the patients with LVEDP $<16 \mathrm{mmHg}$ and patients with LVEDP $\geq 16 \mathrm{mmHg}$ regarding the $\mathrm{MV}$ deceleration time with the $\mathrm{P}$ value $>0.05$. Similarly, study performed by Senechal, et al., ${ }^{10}$ who studied 42 consecutive patients with $\mathrm{AF}$ and found that DT average $(124 \pm 27 \mathrm{~ms})$ did not correlate with PCWP with p-value 0.67.

In contrast, Traversi et al., ${ }^{11}$.studied 51 patients with $\mathrm{AF}$ and heart failure to predict PCWP by Doppler echocardiography and found strong relation between MV deceleration time and elevated PCWP (r.75). This discrepancy in results may be due different type of patient in there study as they studied patients with heart failure.

In our study, the LAVI was statistically significant lower in patient with LVED $<16 \mathrm{mmHg}$ than in patients with $\mathrm{LVEDP} \geq 16 \mathrm{mmHg}(\mathrm{P}$ value $<0.05)$. This was in agreement with Naji, et al., ${ }^{12}$ who studied40 patients with persistent $\mathrm{AF}$ and found higher LAVI in AF group with p-value $<0.001$.

In our study there was statistically significant difference between the two groups with the $\mathrm{P}$ value < 0.05 according to average E/e'. This was in agreement with Wada, et al., ${ }^{13}$ who studied 45 patients (30 men; mean age $69 \pm 9$ years) with chronic $\mathrm{AF}$ for evaluation of LV filling pressure using E/e'in AF patients and found Significant relationships between PCWP and E/e' $(r=0.57, \mathrm{P}<0.001)$.

Also this was in agreement with Kusunose et al., ${ }^{14}$ who studied 56 with chronic AF for evaluation the usefulness of the ratio of $\mathrm{E} / \mathrm{e}$ ' in $\mathrm{AF}$ patients and found that lateral E/e'correlated well with PCWP ( $\mathrm{r}_{\text {_ }}$ $\left.0.74, p_{-} 0.001\right)$.

Also this was in agreement with $\mathrm{Li}$ et al., ${ }^{15}$ who studied 49 patients with non valvular $\mathrm{AF}$ and found that $\mathrm{E} / \mathrm{e}$ ' septal was $10 \pm 3.6$ and $\mathrm{E} / \mathrm{e}$ ' lateral was $8.1 \pm 2.8$ in group A vs. E/e' septal was $14.1 \pm 3.8$ and E/e' lateral was $12 \pm 2.6$ in group B with significant correlation between E/e' and LV filling pressure.

The cutoff value in our study for E/e' is $>14$ and it has Positive Predictive Value $=93.3 \%$ and its Negative Predictive Value $=76 \%$ and the sensitivity to detect elevated LV filling pressure is $70 \%$ and specificity is $95 \%$. 
This was similar to Senechal, et al., ${ }^{10}$ study who studied 42 patients with $\mathrm{AF}$ and determine the sensitivity of E/e' to detect elevated LV filling pressure to be $91 \%$ and specificity $85 \%$.

Also this was in comparable with Kusunose et al., ${ }^{13}$ who studied 56 with chronic AF and determine the sensitivity of E/e' to detect elevated LV filling pressure to be $90 \%$ and specificity $90 \%$.

Our results were different from Wada, et al., ${ }^{12}$ who studied 45 patients with chronic AF for evaluation of LV filling pressure using $\mathrm{E} / \mathrm{e}$ 'in $\mathrm{AF}$ patients and determine the sensitivity of E/e' to detect elevated LV filling pressure to be $50 \%$ and specificity $90 \%$. The low predictive values of this study may be due to smaller number of studied patients of Wada et al., ${ }^{12}$ comparable of our study.

The cutoff value in our study for LAVI is $>34$ $\mathrm{ml} / \mathrm{m} 2$ and it has Positive Predictive Value $=100 \%$ and its Negative Predictive Value $=55.6 \%$ and the sensitivity to detect elevated LV filling pressure is $20 \%$ and specificity is $100 \%$.

This was in agreement with Anderson et al., ${ }^{16}$ who studied 450 patients and found that LAVI has Positive Predictive Value $=91 \%$ and its Negative Predictive Value $=83 \%$ and the sensitivity to detect elevated LV filling pressure is $84 \%$ and specificity is $88 \%$.

The cutoff value in our study for TR velocity is $>2.8$ $\mathrm{m} / \mathrm{sand}$ it has Positive Predictive Value $=63.6 \%$ and its Negative Predictive Value $=55.2 \%$ and the sensitivity to detect elevated LV filling pressure is $35 \%$ and specificity is $80 \%$.

This was lower than Anderson et al., ${ }^{16}$ who found that TR velocity is $>2.8 \mathrm{~m} / \mathrm{s}$ has Positive Predictive Value $=91 \%$ and its Negative Predictive Value $=83 \%$ and the sensitivity to detect elevated LV filling pressure is $84 \%$ and specificity is $88 \%$. This difference in results may be due to their large number of patients comparing to relatively small number of patients in our study.

\section{CONCLUSION}

This study concludes that in patients with AF E/e' was the independent predictor of elevated LVEDP. There is high concordance between E/e' and LVEDP. Average E/e' had modest sensitivity and highest specificity in predicting elevated LVEDP and Diastolic dysfunction.Further larger studies are needed to confirm our finding in different racial groups and clinical subsets.

\section{REFERENCES}

1. Kirchhof P, Benussi S, Kotecha D, et al. ESC Guidelines for the management of atrial fibrillation developed in collaboration with EACTS. Europace; 2016 18:1609-78.

2. KotechaD, and Piccini JP. Atrial fibrillation in heart failure: what should we do? Eur Heart J; 2015 36:3250-7.

3. Kotecha D, Chudasama R, Lane DA, et al. Atrial fibrillation and heart failure due to reduced versus preserved ejection fraction: A systematic review and meta-analysis of death and adverse outcomes. Int J Cardiol. 2016; 203: 660-666.

4. Chiang CE, Naditch-Brule L, Murin J, et al. Distribution and risk profile of paroxysmal, persistent, and permanent atrial fibrillation in routine clinical practice: insight from the real-life global survey evaluating patients with atrial fibrillation international registry. CircArrhythmElectrophysiol. 2012; 5:632639.

5. Tedrow UB, Conen D, Ridker PM, et al. The long- and short-term impact of elevatedbody mass index on the risk of new atrial fibrillation the WHS (Women's Health Study). J Am CollCardiol 2010; 55(21): 2319-2327.

6. Patil VC, Patil HV, Shah KB, et al. Diastolic dysfunction in asymptomatic type 2 diabetes mellitus with normal systolic function. J Cardiovasc Dis Res.2001; 2(4), 213-222.

7. Zile MR, and Brutsaert DL. New concepts in diastolic dysfunction and diastolic heart failure: Part I: diagnosis, prognosis, and measurements of diastolic function. Circulation J, 2002; 105:1387-93.

8. Lang RM, Bierig M, Devereux RB, et al. Chamber Quantification Writing Group; American Society of Echocardiographys Guidelines and Standards Committee; European Association of Echocardiography. Recommendations for chamber quantification: a report from the American Society of Echocardiography's Guidelines and Standards Committee and the Chamber Quantification Writing Group, developed in conjunction with the European Association of Echocardiography, a branch of the European Society of Cardiology. J Am Soc Echocardiogr. 2005; 18:1440-1463.

9. Kotecha D, Mohamed M, Shantsila E, et al. Is echocardiography valid and reproducible in patients with atrial fibrillation? A systematic review. Europace. 2017;19, 1427-1438

10. Senechal M, O’Connor K, Deblois J, et al. A simple Doppler echocardiography method to evaluate pulmonary capillary wedge pressure in patients with atrial fibrillation. Echocardiography. 2008; 25: $57-63$.

11. Traversi E, Cobelli F, Pozzoli M. Doppler echocardiography reliably predicts pulmonary artery wedge pressure in patients 
with chronic heart failure even when atrial fibrillation is present. Eur J Heart Fail. 2001; 3(2) 173-81.

12. Naji F, MihaelPagliaruzzi, Meta Penko, et al. Changes in Left Ventricular Filling in Patients with Persistent Atrial Fibrillation. Int. J. Med. Sci. 2013; 10(13):1876-1879.

13. Wada $Y$, Murata $K$, Tanaka $T$, et al. Simultaneous Doppler tracing of transmitral inflow and mitral annular velocity as an estimate of elevated left ventricular filling pressure in patients with atrial fibrillation. Circulation J. 2012; 76:675-681.

14. Kusunose $K$, Yamada $H$, Nishio $S$, et al. Clinical utility of single-beat E/e' obtained by simultaneous recording of flow and tissue Doppler velocities in atrial fibrillation with preserved systolic function. JACC Cardiovasc Imaging. 2009; 2:1147-1156.

15. Li C, Zhang J, Zhou $\mathrm{C}$, et al.Will simultaneous measurement of E/e' index facilitate the non-invasive assessment of left ventricular filling pressure in patients with non-valvular atrial fibrillation? European Journal of Echocardiography. Eur J Echocardiogr. 2010; 11: 296-301.

16. Andersen OS, Smiseth OA, Dokainish H, et al. Estimating Left Ventricular Filling Pressure by Echocardiography. J Am CollCardiol. 2017; 18; 69(15):1937-1948. 